\title{
Closed-loop control of wavepackets in a free shear-flow
}

\author{
Kenzo Sasaki; Gilles Tissot $\nmid$ André V. G. Cavalieri \\ Flávio Silvestre ${ }^{\S}$ Peter Jordan ${ }^{\top}$ and Damien Biau $\|$
}

\begin{abstract}
This study aims at the attenuation of the unsteady fluctuations along a two-dimensional mixing layer which may be considered as a prototypical problem for the evaluation of estimation and control techniques, and also a canonical problem, when compressibility is considered, for sound radiation by low-Reynolds-number free shear flows. Two strategies are proposed for the estimation of the time evolution of wavepackets based on upstream data of the simulation: a Parabolised-stability-equation (PSE) based transfer function between two positions and an empirical-transfer-function identification technique, which relies on the theoretical background established by the PSE. Both techniques present a similar performance for prediction of the fluctuations between streamwise-separated input and output positions.

Furthermore, the identification method is used to determine the response of the flow to a body force actuation which allows for the elaboration of a Feedforward control framework for the fluctuations via a phase-opposition actuation. This strategy, which is evaluated with three different control laws, presents encouraging results both for the linearized system (i.e. described in terms of transfer functions) and for the non-linear, direct numerical simulation of the mixing layer, in which significant delays of vortex pairing are observed. The established framework is thus seen as a promising technique for real-time flow control aiming at the attenuation of wavepackets, and the corresponding reduction of the radiated sound.
\end{abstract}

\section{Introduction}

The manipulation of flow dynamics through active or passive control represents a challenge for industrial and technological applications. Reduction of drag and consequently of fuel consumption, delay of the transition to turbulence of laminar flows and a reduction of noise levels are some of the foreseeable applications of flow control.

However, there are major obstacles to be overcome prior to the establishment of a feasible control law:

- The inherent non-linearities of the fluid dynamics make it difficult to distill the Navier-Stokes equations to a simplified form that captures the main characteristics of the flow but with a low-computational cost, necessary for the real-time control of fluctuations.

- The high dimensionality of the problem, where each grid point of the discretized system may be treated as a state-variable, making the computational expenses grow rapidly with the domain of calculation.

A promising idea to obtain simplified models to deal with these issues for aeroacoustic applications relates to a description of the most acoustically efficient modes, through a linearization of the Navier-Stokes equations over a mean-flow, which also allows the propagation of each mode or frequency independently. This results in a description of large-scale turbulent structures as wavepackets. Such models, derived in the frequency domain, have been shown to agree with experimental data over the range of frequencies

*PhD Student, Instituto Tecnológico de Aeronáutica, São José dos Campos, Brazil.

†Post-doctoral Student, Instituto Tecnológico de Aeronáutica, São José dos Campos, Brazil.

¥Professor, Instituto Tecnológico de Aeronáutica, São José dos Campos, Brazil.

$\S$ Professor, Instituto Tecnológico de Aeronáutica, São José dos Campos, Brazil.

ף Research Scientist, Institut Pprime, CNRS- University of Poitiers-ENSMA, Poitiers, France.

"École Nationale Supérieure d'arts et Métiers, Paris, France 
$0.3 \leq \mathrm{St} \leq 0.9$ and azimuthal numbers $m=0$ and $1 .{ }^{1-3}$ These large-scale structures are characterized by their low azimuthal number and coherence over the radial and axial directions which make them dominate the acoustic radiation, particularly at shallow angles to the jet axis.

For real-time closed-loop control, frequency-domain models do not suffice. One must be able to predict in the time domain, from given limited flow measurements, how the perturbations will evolve downstream. This task is complicated by the fact that, wavepackets are not harmonic (in the case of a turbulent jet, for instance, the wavepackets are forced by the flow within the nozzle, which has a turbulent boundary layer).

In our previous effort ${ }^{4}$ we made use of time-domain transfer functions in order to educe real-time SISO (Single-Input-Single-Output) models for a turbulent jet. These models were based on the near-field pressure measurements of ${ }^{5}$ and on a first-principles method relying on the Parabolized Stability Equations (PSE). The frequency-domain models mentioned earlier allowed for the construction of a transfer function between a single position upstream and one or several positions downstream (with the second case corresponding to a Multiple-Outputs model - SIMO).

In the first case, an empirical transfer function was built from the identification of unsteady measurements in the flow. The second case corresponds to a first-principles-based model, built from PSE. These models, when inverse Fourier transformed, provide a time-domain transfer function which, when convolved with pressure measurements in the vicinity of the exit plane permit the prediction of the entire flow-field downstream, as presented in figure 1.

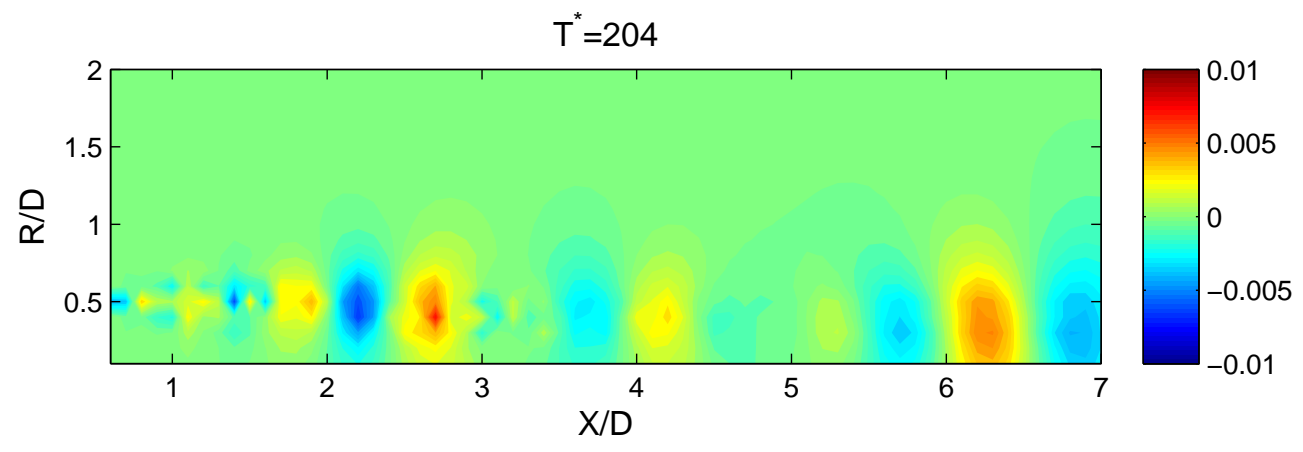

Figure 1. PSE transfer function pressure field prediction obtained from measurements along the $\frac{X}{D}=0.5$ ring cylindrical coordinates were considered, with $\frac{R}{D}$ defining the radial distance from the jet axis. Further details may be found on. ${ }^{4}$

The good performance of these models (figure 2), when compared to experimental measurements of a high-Reynolds turbulent jet, combined with their low computational cost, make them suitable candidates for closed-loop controlled systems.

In this work, we apply the previously derived methods to a simpler problem, a two-dimensional incompressible mixing layer, for which a DNS solver can be used to implement closed-loop control. The 2D mixing-layer is a canonical problem of sound generation by low-Reynolds free-shear flows, ${ }^{6,7}$ where vortex pairing has been detected as the main sound-generation mechanism. With the present incompressible DNS it is not possible to evaluate directly sound radiation; however, the present simulation allows us to evaluate if the controller is capable of delaying or suppressing vortex pairing in the mixing layer, which in turn should lead to considerable reductions of the radiated sound. We derive a control law for this system which is based on the empirical transfer function formalism combined with the PSE theoretical model.

This paper is organized as follows. The problem we consider is described in section II, the empirical and PSE transfer functions are described in section III and the control laws are presented in section IV. Finally, the results and a comparison of the techniques for the closed-loop controlled system are presented in section $\mathrm{V}$ with our conclusions and foreseeable future work presented in section VI.

\section{Numerical simulation}

The direct numerical simulation (DNS) solves the incompressible bi-dimensional Navier-Stokes equations using a spectral method. The streamwise direction is discretised using $N_{x}=512$ Fourier modes and the 

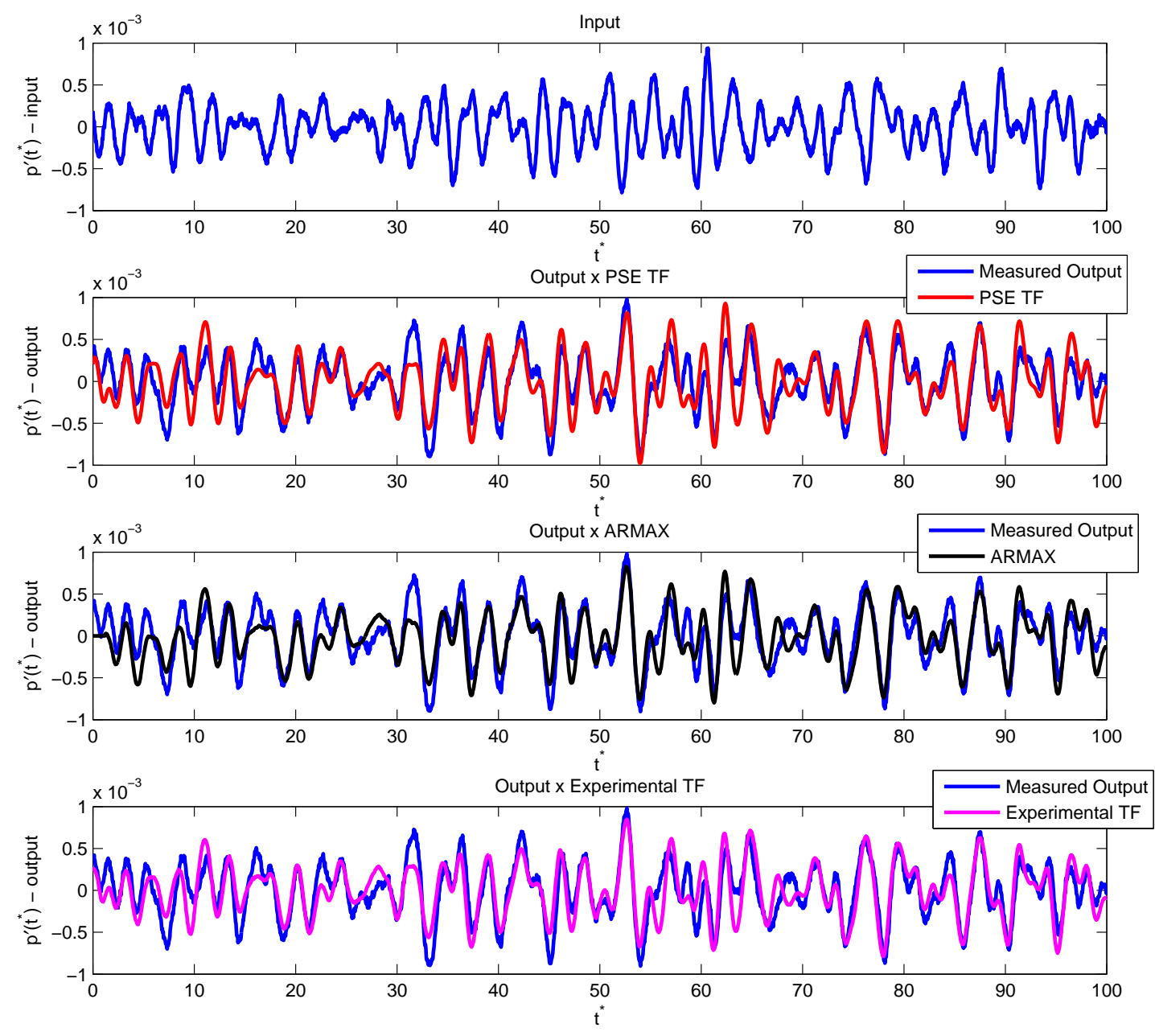

Figure 2. Comparison between measured non-dimensional pressure fluctuations, PSE transfer function, empirical transfer function and ARMAX predictions, respectively, as presented on, ${ }^{4}$ for input at 1.3 and output at 2.1 diameters from the jet nozzle.

transversal direction using $N_{y}=70$ Chebyshev polynomials. The domain is $x=\left[0: L_{x}\right], y=\left[-y_{\text {inf }}: y_{\text {inf }}\right]$ with $L_{x}=600$, and $y_{\text {inf }}=200$. The simulation non-dimensional time step is $\mathrm{d} t=0.02$, where solutions are stored every $\mathrm{d} t_{\text {save }}=0.4$ for a time $T=2000$. The inflow condition is specified as

$$
U_{b}(y)=\frac{1}{2}\left(1+\tanh \left(\frac{y}{2}\right)\right)
$$

plus some unsteady perturbations defined by random variables for the control, as presented on section IV. The inflow (1) imposes a unitary velocity difference across the shear $\Delta U$ and a unitary vorticity and momentum thickness. The Reynolds number used is $R e=\frac{\Delta U \delta_{\omega}}{\nu}=100$, where $\nu$ is the kinematic viscosity and $\delta_{\omega}$ is the vorticity thickness.

Because we are using a Fourier decomposition in the non-homogeneous streamwise direction, a sponge zone is applied at the end of the domain, so that the solution $q$ reached the inflow state $q_{i} n$. It consists of adding a forcing term $\boldsymbol{f}=-\lambda\left(\boldsymbol{q}-\boldsymbol{q}_{\text {in }}\right)$ with $\lambda=\frac{20}{2}\left(1+\tanh \left(\frac{x-0.85 L_{x}}{10}\right)\right)$. Figure 3 shows an instantaneous vorticity field of the mixing layer simulation used for the PSE validation. 


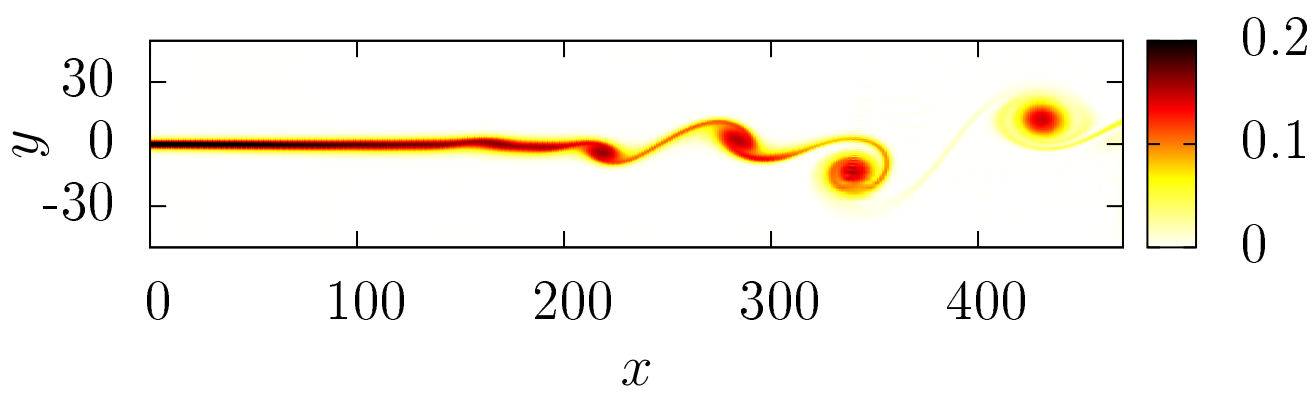

Figure 3. Instantaneous vorticity field of the mixing layer DNS.

\section{Estimation techniques}

\section{III..1. 2D PSE transfer function}

By following a similar strategy to that developed previously in ${ }^{4}$ the linearity of the PSE may be exploited in order to obtain a Single-Input-Single-Output model between any given pair of positions along the flow and considering any of the dependent variables (pressure or the vertical and horizontal velocities, for this particular problem).

Defining the complex quantities $R(\omega)$ and $Y(\omega)$ of two positions along the flow considered respectively as an input and an output, with $\omega$ defining the angular frequency, and assuming a linear relation between these, a frequency-domain transfer function may be written as:

$$
G(\omega)=\frac{Y(\omega)}{R(\omega)} .
$$

From which a time-domain transfer function is readily available from the inverse Fourier transform of $G(\omega)$

$$
g(t)=\frac{1}{2 \pi} \int_{-\infty}^{\infty} G(\omega) e^{-i \omega t} \mathrm{~d} \omega .
$$

From $g(t)$, the output $y(t)$ may be estimated from measurements of the input $r(t)$, which defines a SISO system as:

$$
y(t)=\int_{0}^{\infty} g(\tau) r(t-\tau) \mathrm{d} \tau .
$$

Only the behaviour of linear wavepackets is predicted with equation (4) and a deterioration of the comparisons between such prediction and the actual outputs of the DNS is expected as one moves downstream.

PSE was used to calculate the fluctuations repeatedly for a range of Strouhal numbers, which is feasible due to the low computational cost of this method.

The frequency and time-domain transfer functions obtained from this method along with their prediction compared to the DNS output are presented in figure 4, considering the streamwise velocity component in the pair $\left(X_{1}, Y_{1}\right)=(75,1)$ and $\left(X_{2}, Y_{2}\right)=(100,1)$ for the definition of the transfer function. The close agreement between these two signals validates the method. The time at which the peak is observed for the transfer function $g(t)$ corresponds to the average convective time which is taken to the perturbation to travel between input and output positions.

\section{III..2. System identification - empirical transfer function}

Following a similar procedure to that outlined $\mathrm{in}^{4}$ it is possible to build an empirical transfer function with inflow disturbances as broadband perturbations.

For this case, when assuming a linear relation between the variables at different axial locations, it is possible to define a frequency domain transfer function which considers the auto and cross spectral densities of the input and output signals ( $S_{r r}$ and $S_{r y}$, respectively), 

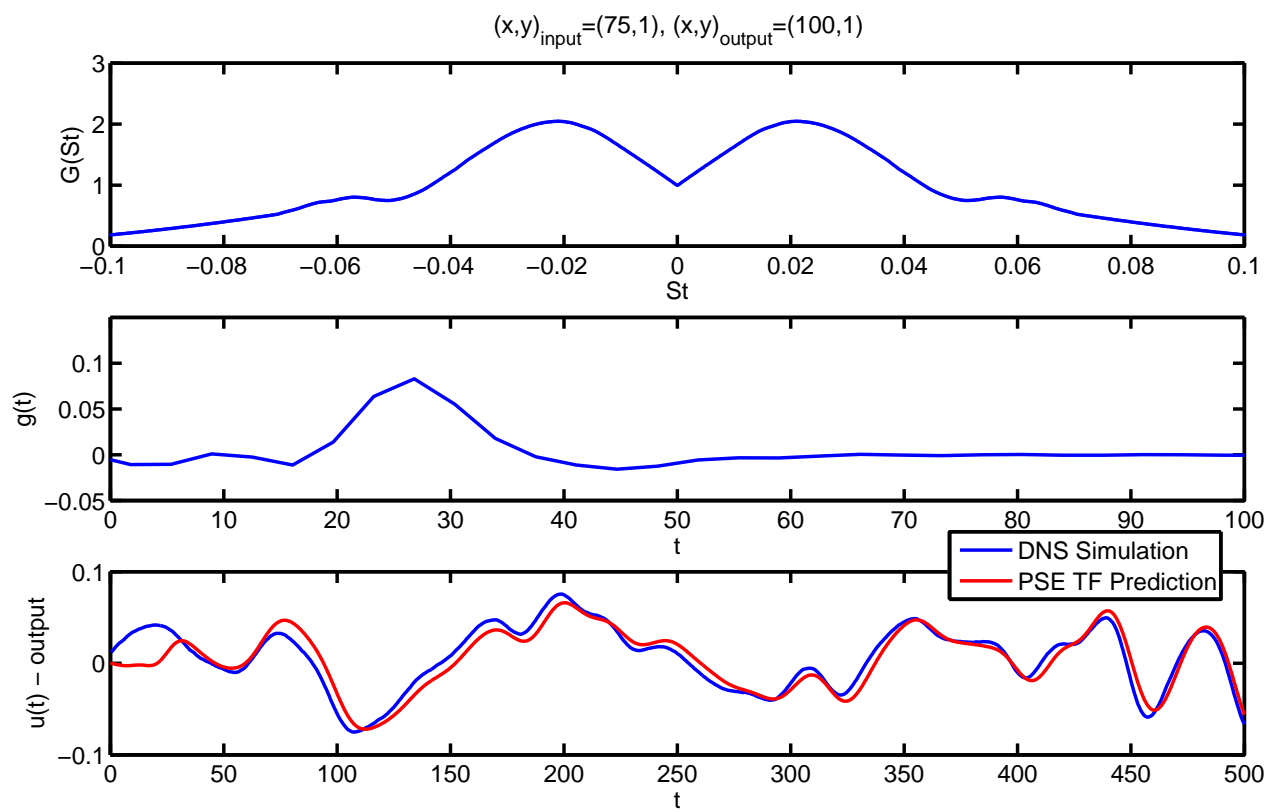

Figure 4. PSE frequency and time-domain transfer functions applied to the 2D viscous incompressible problem and comparison of the predictions against a DNS simulation for the $u(t)$ streamwise velocity component.

$$
G(\omega)=\frac{S_{r y}(\omega)}{S_{r r}(\omega)}
$$

Equation 5 may be understood as the optimal response of the system in the least squares formalism. ${ }^{8}$ Even though this corresponds to an identification using the unsteady simulation, it still comprises a formulation which is consistent with the linear framework established by the PSE analysis. From equation (5), a timedomain model is obtained from the inverse Fourier transform by following the same procedure as that outlined in III..1, which will allow the prediction of the output.

Figure 5 presents the resultant frequency and time-domain transfer functions, and the comparison of such prediction to the data coming from the simulation, for the pair $\left(X_{1}, Y_{1}\right)=(75,1)$ and $\left(X_{2}, Y_{2}\right)=(100,1)$. A similar result to that obtained from the PSE transfer-function prediction is obtained, which also validates this method.

\section{Control law}

The control of the fluctuations is based on a body force in the $x$ or $y$ directions, $f(x, y, t)$ which is inserted at a given position in the flow and causes a perturbation to the velocity fluctuations. Such a perturbation seeks to represent the effect of an actuator at that given position, e.g. microjets in the velocity field of the mixing layer. Equation (6) translates this idea for the bidimensional case, where $\left(x_{2}, y_{2}\right)$ denotes the actuator position, $L$ will determine the spatial support of the applied body force, and $a(t)$ gives the time behaviour of the perturbation.

$$
f(x, y, t)=a(t) e^{-\frac{\left(x-x_{2}\right)^{2}}{L^{2}}} e^{-\frac{\left(y-y_{2}\right)^{2}}{L^{2}}}
$$

Once the perturbation has been defined, the transfer function which models the actuation $\left(G_{A}(\omega)\right)$ is determined by the same procedure outlined in Section III..2. In order to obtain $G_{A}(\omega)$, the inflow conditions are considered to be null, $a(t)$ is set to be a broadband signal and the identification is performed between the time signal $a(t)$ and the resulting fluctuations further downstream. Figure 6 presents the validation of the empirical-transfer-function approach applied to a perturbation in the axial direction.

With the control and estimation transfer functions, a control framework may consider a feedforward strategy which relates to the convective nature of the flow under study. Such approach was followed by ${ }^{9}$ for a backward-facing step, using estimation and control models identified through ARMAX. In order to obtain 

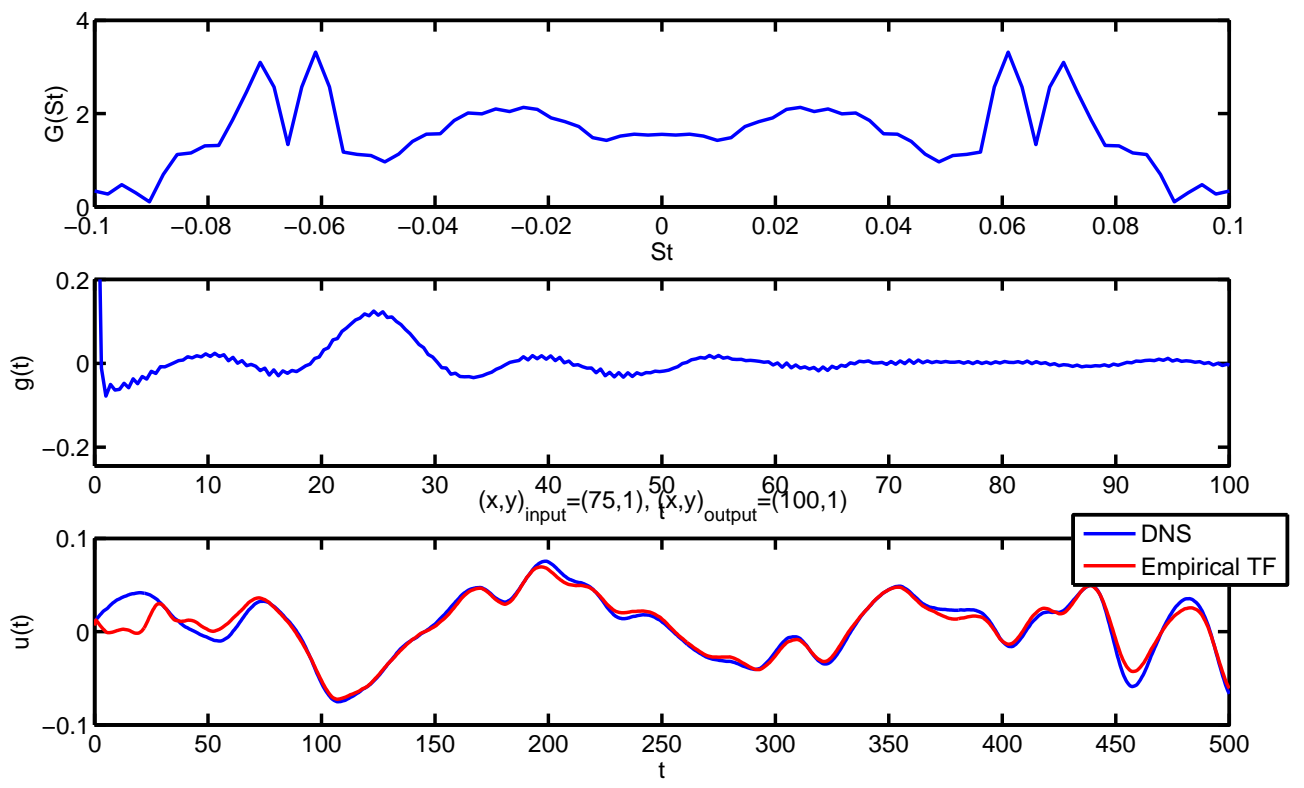

Figure 5. Empirical frequency and time-domain transfer functions applied to the 2D viscous incompressible problem and comparison of the predictions against a DNS simulation for the axial-velocity component.

that, previous measurements will be used to define an actuation, seeking to eliminate the fluctuations at a downstream position. It is thus useful to define the following positions:

- $\left(X_{1}, Y_{1}\right)$ defines the input position, where the fluctuations are assumed to be measured. The resulting time, and frequency domain signals are, respectively $i(t)$ and $I(\omega)$.

- $\left(X_{2}, Y_{2}\right)$ refers to the position where the actuation will occur; its time and frequency signal are, respectively $a(t)$ and $A(\omega)$, with the spatial support defined in equation 6 .

- The output $(o(t)$ or $O(\omega))$ is measured at $\left(X_{3}, Y_{3}\right)$, downstream of the actuation.

Therefore, it is useful to define two sets of transfer functions; $G_{I_{k}}$ represents the estimation between input and position $\left(x_{k}, y_{k}\right)$, and $G_{A}$ is the actuation transfer function between the actuator, at $\left(X_{2}, Y_{2}\right)$ and the output.

The output signal is considered to be obtained via a superposition of the undisturbed (open-loop) and actuation signals, leading to a closed-loop control scheme.

$$
O(\omega)=I(\omega) G_{I 3}(\omega)+A(\omega) G_{A}(\omega)
$$

With these definitions, three control strategies, which seek to minimize the output via different laws were evaluated, and will be presented in sections IV.A and IV.B.

\section{IV.A. Proportional and Proportional-Integral}

For the Proportional (P) and Proportional-Integral (PI) cases, the actuation is set to be a sum of constants multiplied by estimates of the signal at positions around the actuator, at position 2 , this has the objective of increasing the degrees of freedom of the controlled, since each gain is a scalar value, which is not frequencydependent. In the frequency domain this is may be written as in equation 8 .

$$
O(\omega)=I(\omega) G_{I 3}(\omega)+\sum_{i=1}^{N} K_{p i} G_{I 2}^{i} I(\omega)
$$



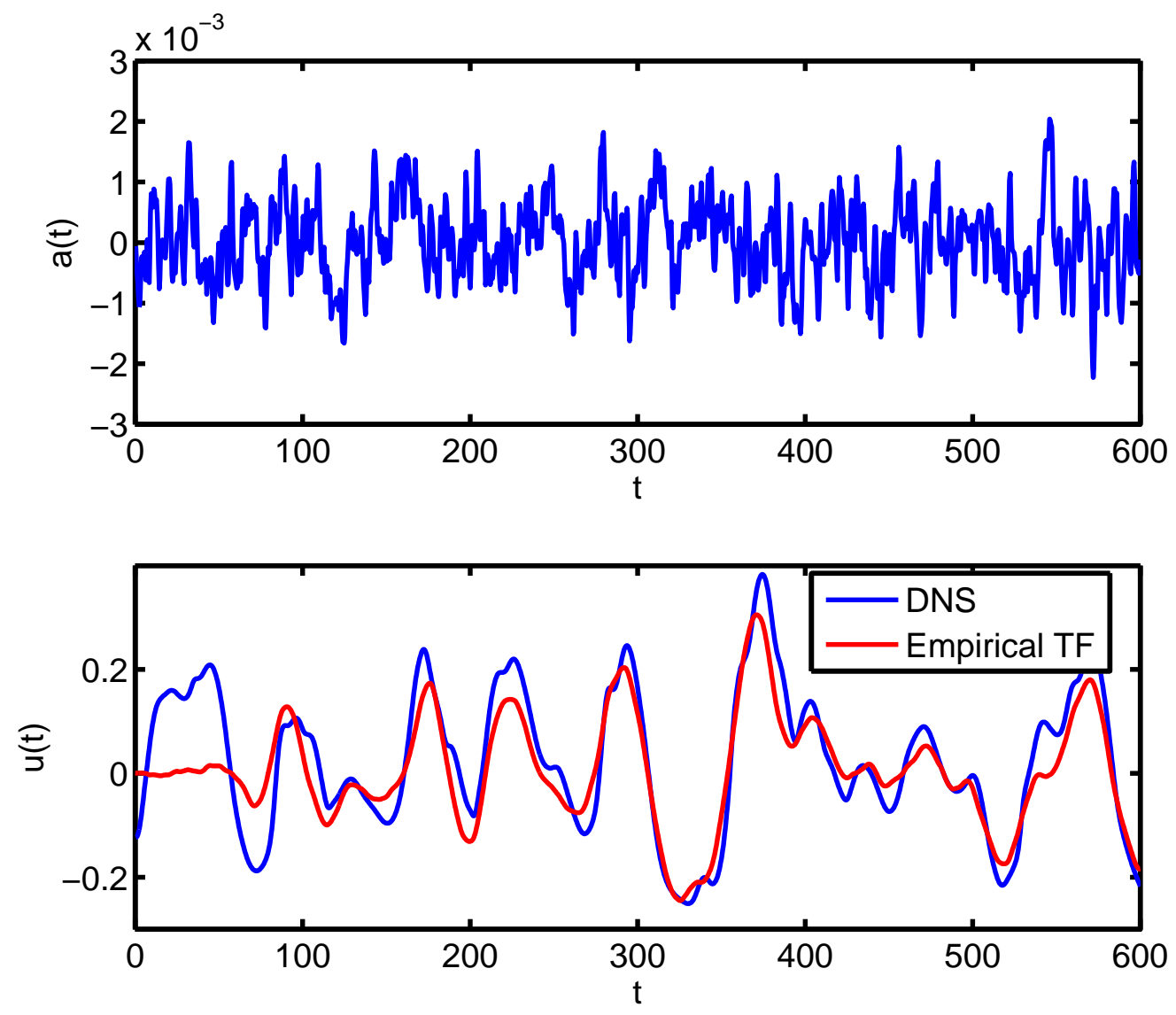

Figure 6. Actuation $a(t)$ and predicted resulting behaviour of the axial velocity fluctuations compared to the actual simulation.

For the results of the following sections, the number of estimation positions will be always equal to three, both for the proportional and proportional-integral cases. Therefore, if the output is to be minimized, we are solving the following equation, in the frequency domain, for the proportional case;

$$
K_{p 1} G_{I 2}^{1}+K_{p 2} G_{I 2}^{2}+K_{p 3} G_{I 2}^{3}=-\frac{G_{I 3}(\omega)}{G_{A}(\omega)}
$$

Where $G_{I 2}^{j}$ denotes transfer functions between the input and position $j$, around the actuator. Equation 9 may be solved in the frequency domain. Since the gains are defined as scalar values, such strategy will not lead $O(\omega)$ to be zero and the optimal gains may be found via Moore-Penrose pseudoinverse. An alternative procedure is to determine the optimal gains already in the time-domain, aiming at minimizing $o(t)$.

Writing equation 8 in the time-domain, for the proportional case;

$$
o(t)=\int_{t}^{t+\tau} i(\tau) g_{i 3}(t-\tau) d \tau+\sum_{i=1}^{N} k_{p i}\left[\int_{t}^{t+\tau} i(\tau)\left(g_{i 2}^{i}\right)(t-\tau) d \tau\right] \star g_{a}(t)
$$

When the integral gain is considered, the output is obtained analogously, with a second set of gains added to include the integral of the estimation signals around the actuator. This is defined in equation 11.

$o(t)=\int_{t}^{t+\tau} i(\tau) g_{i 3}(t-\tau) d \tau+\sum_{i=1}^{N} k_{p i}\left[\int_{t}^{t+\tau} i(\tau)\left(g_{i 2}^{i}\right)(t-\tau) d \tau\right] \star g_{a}(t)+\sum_{i=1}^{N} k_{i i}\left[\int_{t=0}^{t=T_{0}}\left[\int_{t}^{t+\tau} i(\tau)\left(g_{i 2}^{i}\right)(t-\tau) d \tau\right] d t\right] \star g_{a}(t)$

where $k_{i i}$ define the $\mathrm{i}$-th integral gain and $T_{0}$ is the buffer to calculate the integral numerically. 
Finally, in order to avoid obtaining an actuation which is too high in amplitude, a term to penalize the control signal was added to the optimization scheme described in equations 10 and 11.

$$
J=\int_{0}^{T}\left(o(t)^{2}+R a(t)^{2}\right) \mathrm{d} t
$$

The index $J$ is then minimized using the proportional or proportional-integral gains. The following results for the P and PI controllers consider such optimization scheme, performed in the time-domain.

\section{IV.B. System Inversion}

An alternative method is to define the actuation to be a frequency-dependent gain multiplied by the input, i.e. $A(\omega)=-K(\omega) I(\omega)$. Therefore, the output will be zero if $K(\omega)$ is of the form:

$$
K(\omega)=\frac{G_{I}(\omega)}{G_{A}(\omega)},
$$

and the actuation will be given, in the time-domain, as a convolution with the input:

$$
a(t)=\int_{-T}^{T} k(\tau) i(t-\tau) \mathrm{d} \tau,
$$

where $k(t)$ is the inverse Fourier transform of $K(\omega)$.

If the system is causal, the integral in equation (14) is expected to be taken from 0 to $T$. Furthermore, $T$ will define the number of samples to be stored before the actuation starts. In practical terms, it corresponds to the length of the numerically calculated $k(t)$.

When performing the inversion given in equation 13 it should be noted that only frequency regions where the linear model is able to reproduce the dynamics of the non-linear system may be considered. This is a requirement of a feed-forward control law, such that noise present at frequencies where the linear model is not representative of the real system are not amplified, leading to a destabilization of the plant. ${ }^{10}$

Such a technique, which relies on an inversion of the system, such that the actuation is set to eliminate the output signal, results in a gain which is a function of the considered frequency, presenting a greater number of degrees of freedom, when compared to the previous, proportional and proportional-integral cases. 


\section{Results}

\section{V..1. Linearized system}

Prior to the implementation of the control law on the DNS, the calculated gains were validated using the linearized system i.e., considering the formulation based on the transfer functions. For this case, equation (7) is implemented and the axial velocity is considered as the control variable to be minimized. Actuation is here taken as a body force in the $x$ direction.

Figures 7, 8 and 9 present the open-loop prediction (uncontrolled) signal compared to the controlled case, obtained from the linearized system via the system inversion, proportional and proportional integral control laws.

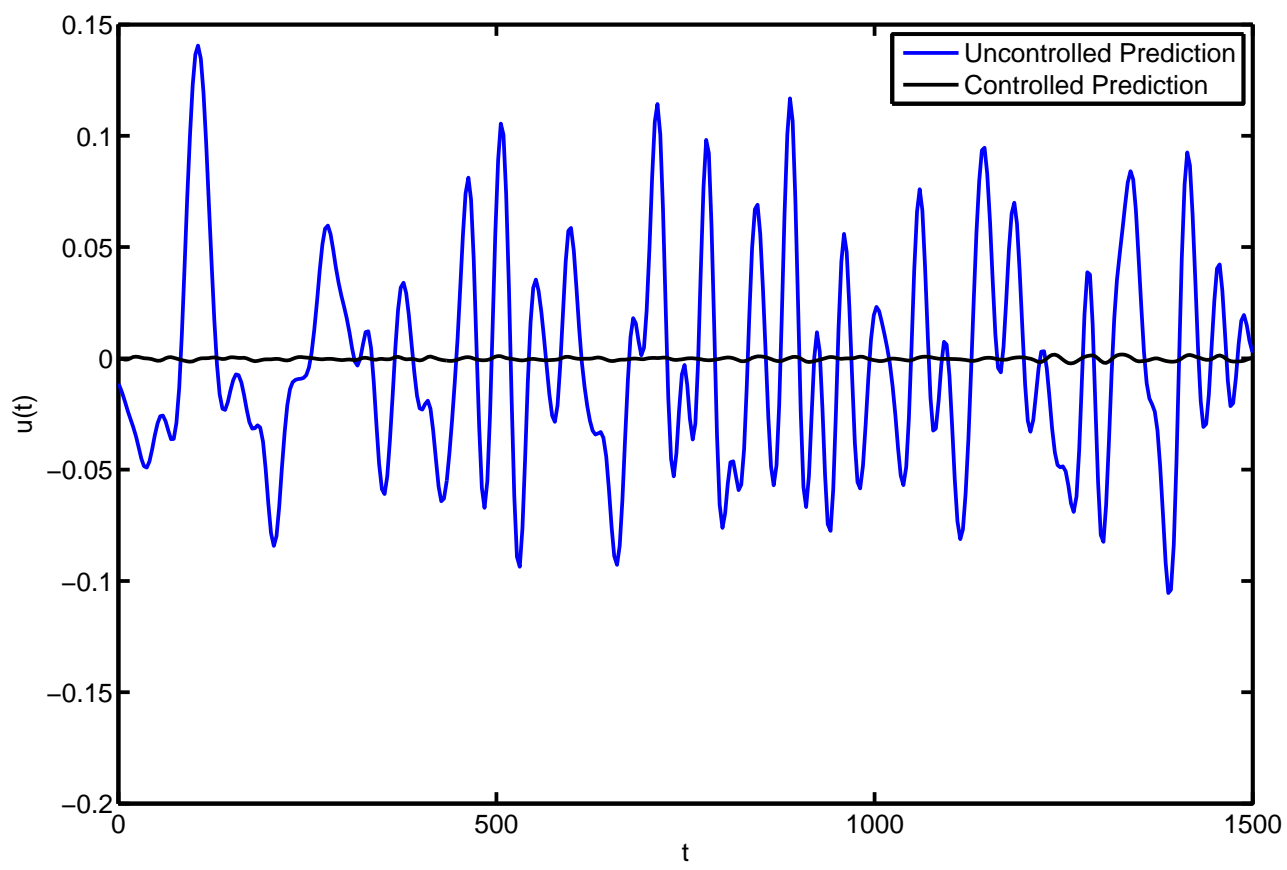

Figure 7. Open-loop prediction obtained from the PSE transfer function and the controlled case via system inversion, considering the linear control framework, with the objective of minimizing the axial-velocity component.

For the three cases, the control is seen to be effective in reducing the amplitude of the fluctuations. However, due to the lower number of degrees of freedom of the P and PI controllers, when compared with that obtained directly via system inversion, the output is not zero.

With this validation performed using the linearized system, we seek to implement the same control law on the DNS.

\section{V..2. DNS results}

The control laws derived in section IV were then implemented on the DNS which contains non-linear interactions absent in the linearised models, and hence constitutes on a more challenging problem for the derived control law. The implementation considered a buffer size of two hundred timesteps and a discretization in time for the control gains $k(\tau)$ which was fine enough to capture the most unstable frequencies.

Figure 10 presents the results of the control action via system inversion, via a body force positioned either along the axial or transverse directions, aiming at reductions of the axial and transverse velocity components, respectively. The position of the objective was $(X, Y)=(125,0)$.

Although for each control scheme the objective was at either axial or transverse velocity components separately, the actuation presents the beneficial effect of reducing both components. Figure 11 presents the comparison of the turbulent kinetic energy of the three cases evaluated in figure 10 and shows that the reductions remain throughout the flow, downstream of the actuation. Furthermore, using a body force 


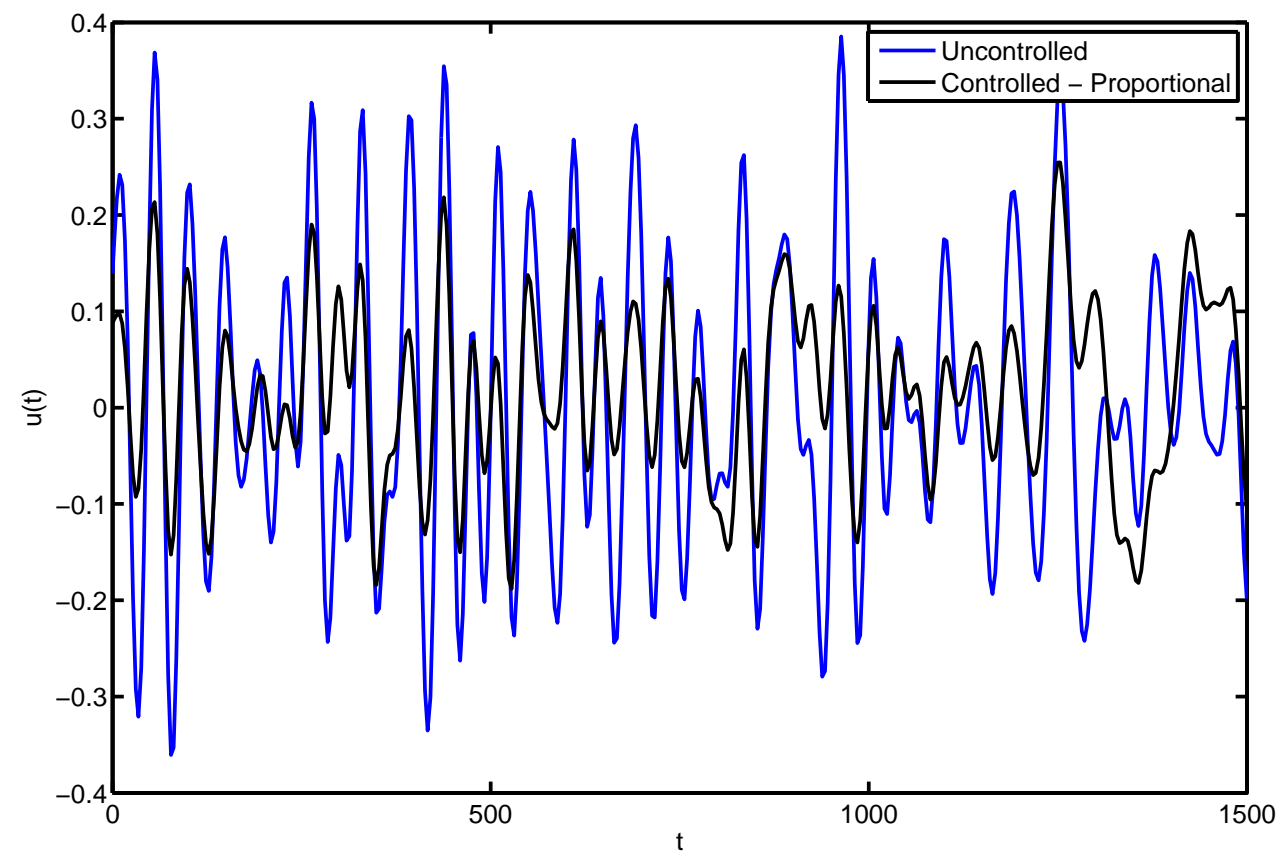

Figure 8. Open-loop prediction obtained from the PSE transfer function and the controlled case via proportional law, considering the linear control framework, with the axial velocity fluctuation as the control objective.

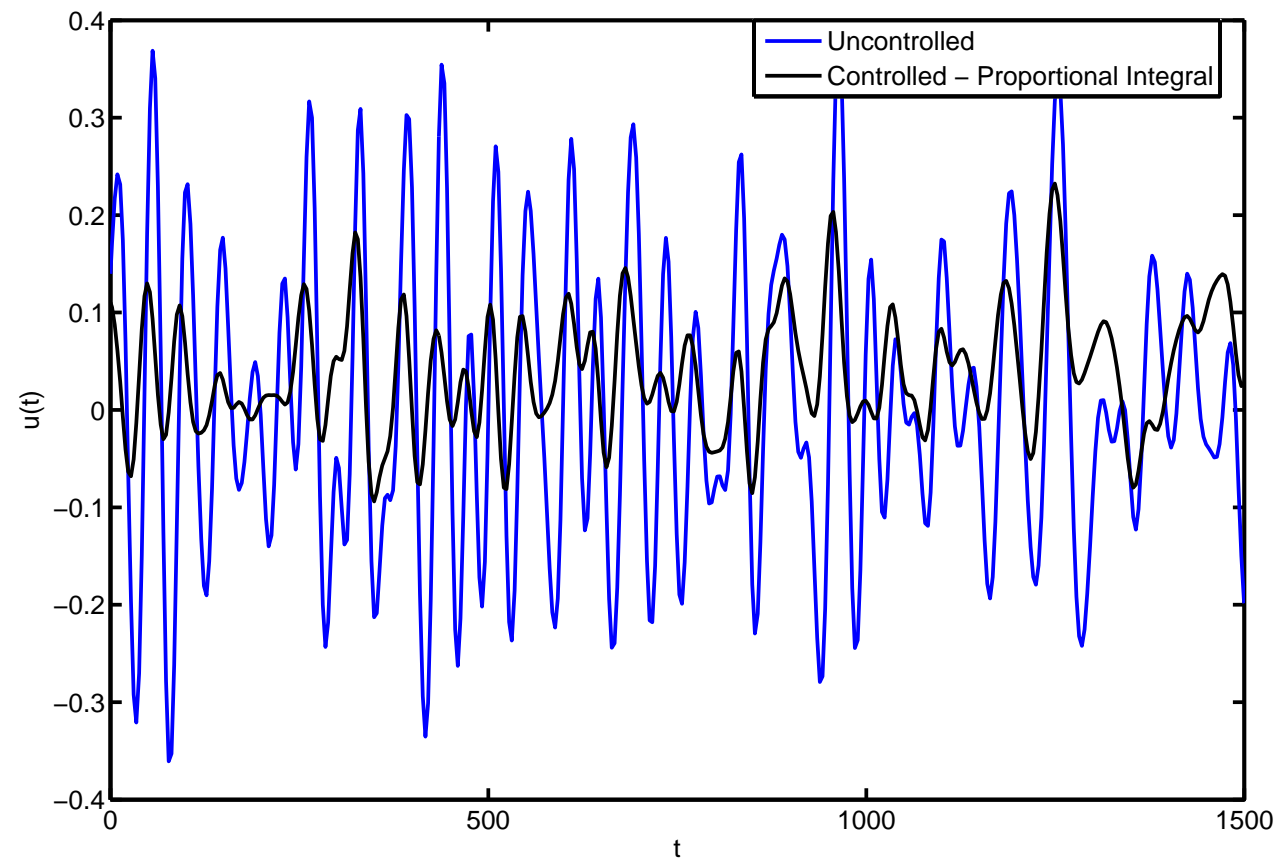

Figure 9. Open-loop prediction obtained from the PSE transfer function and the controlled case via proportional integral law, considering the linear control framework, with the axial velocity fluctuation as the control objective.

on the y-axis appears to be more efficient, at least at the position of the objective. The Kelvin-Helmholtz instability of the mixing layer leads to significant growth rates, which are not cancelled by the actuation; however, significant decreases of amplitude are obtained in downstream stations, with TKE reductions of up 

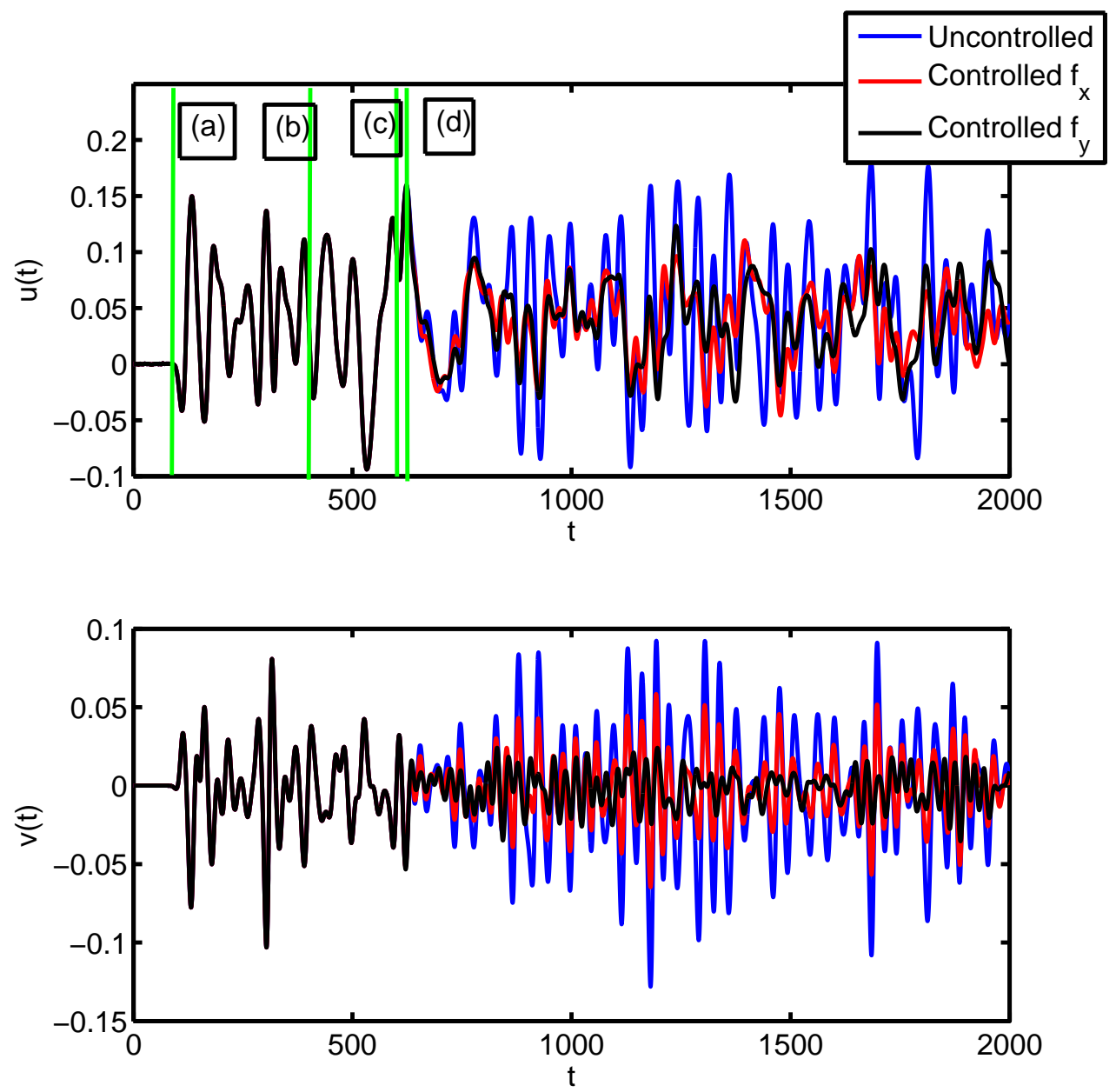

Figure 10. Comparison of the uncontrolled simulation against the controlled case, using system inversion, for the axial and transverse velocity fluctuations, with the time to the fluctuation to reach the actuator (a), transient (b), buffer (c) and time to reach the actuation (d), highlighted.

to $50 \%$.

Figure 12 presents the resulting turbulent kinetic energy of the flow when it is controlled with a proportional or proportional-integral control law and their comparison against the system inversion method. We observe that as the number of degrees of freedom of the control law increases (from proportional, proportionalintegral to system inversion), the consequent reductions of the amplitude of the fluctuations is greater, which is reflected on the turbulent kinetic energy of the flow.

Finally, the vorticity fluctuations of the uncontrolled and system inversion controlled cases are presented in figure 13. As the control action takes place, vortex pairing is delayed. This supplies evidence that for the analogous compressible mixing layer problem ${ }^{6,7,11}$ or low Reynolds number jet ${ }^{12,13}$ the control action would result in lower sound radiation.

\section{Conclusions}

Two transfer-function-based strategies for the real-time prediction of mixing-layer fluctuations were evaluated. In spite of the fact that they present different levels of empiricism, they performed equally well, for the prediction of the velocity and pressure fluctuations in a turbulent mixing layer (although not shown, similar results were obtained for the pressure fluctuations).

Furthermore, the determination of how broadband perturbation affects the flow was obtained via an empirical transfer function technique based on the PSE theoretical background. Such a transfer function 


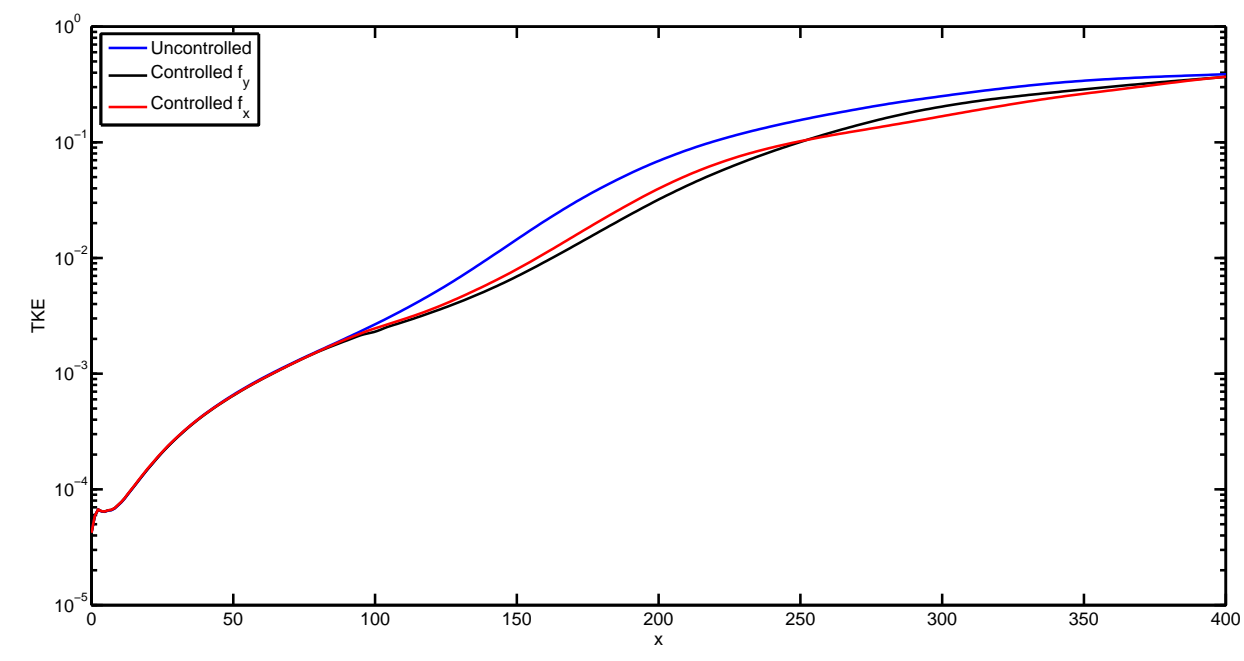

Figure 11. Turbulent kinetic energy for the uncontrolled and controlled simulations via single body force, using system inversion.

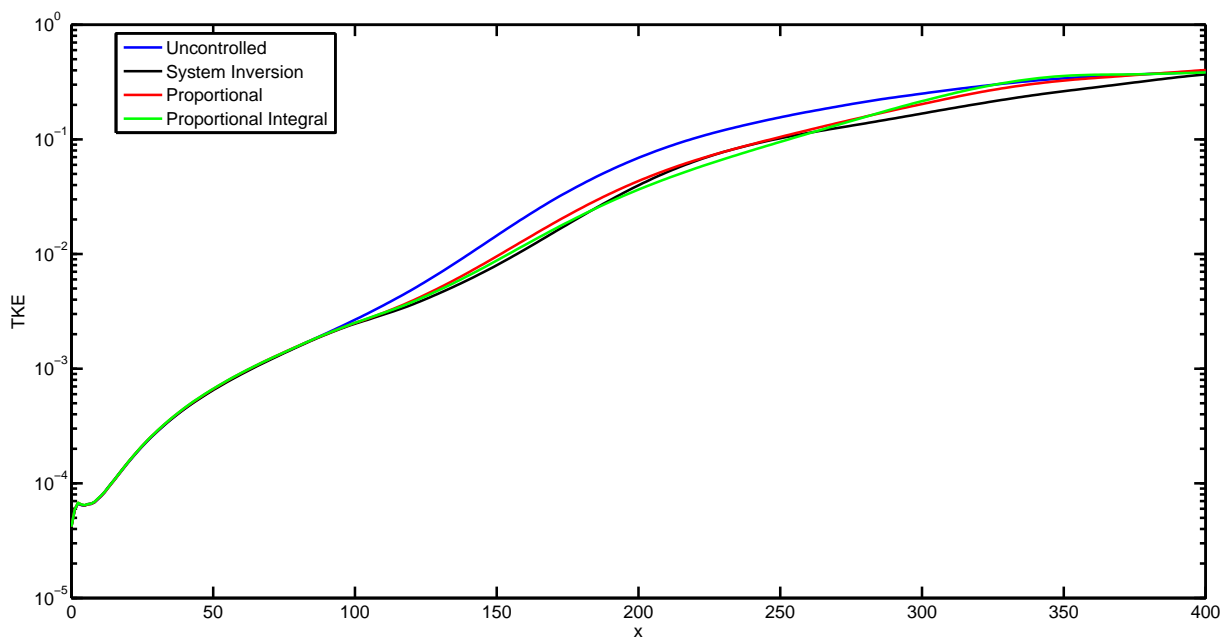

Figure 12. Turbulent kinetic energy for the uncontrolled and controlled simulations via single body force, comparison of the three control methodologies.

allows the establishment of a Feedforward control framework with the objective of attenuating the amplitude of the fluctuations at a given output position. The performance of this method has been validated for three different control-laws, both for linear and non-linear simulations. The differences in the performance of the control laws are understood in terms of the number of degrees of freedom of the controllers. Controllers were able to significantly delay vortex roll-up and pairing, which is expected to have an impact on sound radiation by low-Reynolds flows.

\section{Acknowledgements}

K. Sasaki acknowledges the financial support via Casemiro Montenegro foundation, project ITA-MP. 


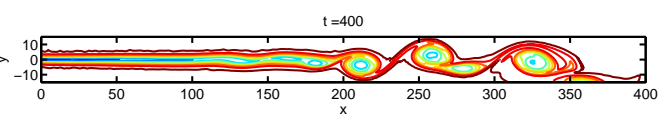

(a)

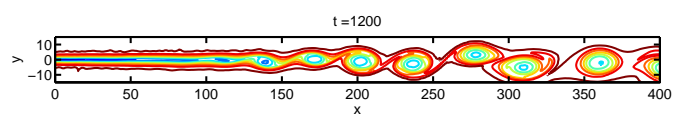

(c)

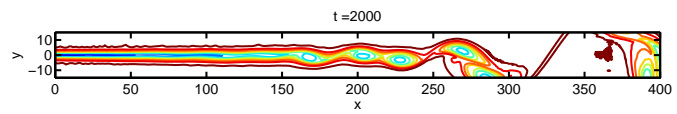

(e)

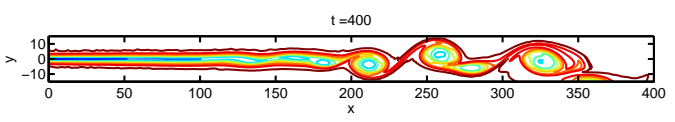

(b)

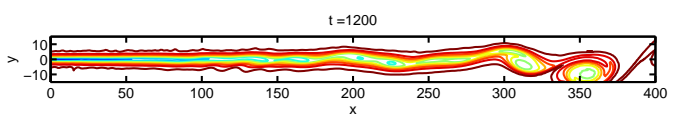

(d)

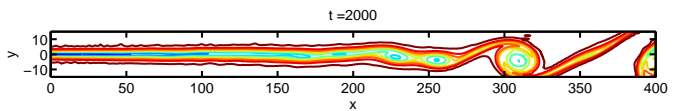

(f)

Figure 13. Comparison of the vorticity fluctuations for the uncontrolled (left) and controlled (right) cases. The delay in the vortex pairing becomes apparent..

\section{References}

${ }^{1}$ Cavalieri, A. V., Rodríguez, D., Jordan, P., Colonius, T., and Gervais, Y., "Wavepackets in the velocity field of turbulent jets," Journal of Fluid Mechanics, Vol. 730, 2013, pp. 559-592.

${ }^{2}$ Jordan, P. and Colonius, T., "Wave packets and turbulent jet noise," Annual Review of Fluid Mechanics, Vol. 45, 2013, pp. $173-195$.

${ }^{3}$ Rodríguez, D., Cavalieri, A. V. G., Colonius, T., and Jordan, P., "A study of linear wavepacket models for subsonic turbulent jets using local eigenmode decomposition of PIV data," European Journal of Mechanics-B/Fluids, 2014.

${ }^{4}$ Sasaki, K., Piantanida, S., Cavalieri, A. V., and Jordan, P., "Real-time modelling of wavepackets in turbulent jets," $21 s t$ AIAA/CEAS Aeroacoustics Conference, 2015, p. 2214.

${ }^{5}$ Breakey, D. E., Jordan, P., Cavalieri, A. V., Léon, O., Zhang, M., Lehnasch, G., Colonius, T., and RODRiguez, D., "Near-field wavepackets and the far-field sound of a subsonic jet," 19th AIAA/CEAS Aeroacoustics Conference, AIAA Paper, Vol. 2083, 2013.

${ }^{6}$ Colonius, T., Lele, S. K., and Moin, P., "Sound generation in a mixing layer," Journal of Fluid Mechanics, Vol. 330, 1997, pp. 375-409.

${ }^{7}$ Wei, M. and Freund, J. B., "A noise-controlled free shear flow," Journal of Fluid Mechanics, Vol. 546, 2006, pp. 123-152.

${ }^{8}$ Bendat, J. S. and Piersol, A. G., Random data: analysis and measurement procedures, Vol. 729, John Wiley \& Sons, 2011.

${ }^{9}$ Hervé, A., Sipp, D., Schmid, P. J., and Samuelides, M., "A physics-based approach to flow control using system identification," Journal of Fluid Mechanics, Vol. 702, 2012, pp. 26-58.

${ }^{10}$ Devasia, S., "Should model-based inverse inputs be used as feedforward under plant uncertainty?" Automatic Control, IEEE Transactions on, Vol. 47, No. 11, 2002, pp. 1865-1871.

${ }^{11}$ Cheung, L. C. and Lele, S. K., "Linear and nonlinear processes in two-dimensional mixing layer dynamics and sound radiation," Journal of Fluid Mechanics, Vol. 625, 2009, pp. 321-351.

${ }^{12}$ Mitchell, B. E., Lele, S. K., and Moin, P., "Direct computation of the sound generated by vortex pairing in an axisymmetric jet," Journal of Fluid Mechanics, Vol. 383, 1999, pp. 113-142.

${ }^{13}$ Violato, D. and Scarano, F., "Three-dimensional vortex analysis and aeroacoustic source characterization of jet core breakdown," Physics of Fluids (1994-present), Vol. 25, No. 1, 2013, pp. 015112. 